\title{
Research on Ship Trajectory Tracking with High Precision Based on LOS
}

\author{
Liu Hengzhi ${ }^{1}$, Geng Tao $^{2}$, Zhang Wei ${ }^{3}$ \\ ${ }^{1}$ College of Physics and Electronics, Henan University, Henan Kaifeng 475004, China \\ ${ }^{2}$ College of Physics and Electronics, Henan University, Henan Kaifeng 475004, China \\ ${ }^{3}$ Henan Academy of Science Applied Physics Institute Co.,Ltd,Henan Zhengzhou450000, China
}

\begin{abstract}
Aiming at how precise to track by LOS, a method is proposed. The method combines the advantages of LOS simplicity and intuition, easy parameter setting and good convergence, with the features of GPC softening, multi-step prediction, rolling optimization and excellent controllability and robustness. In order to verify the effectiveness of the method, the method is simulated by Matlab. The simulation's results show that it makes ship tracking highly precise.
\end{abstract}

\section{Introduction}

The role of oceans is very important for humans. On the one hand, the ocean is related to the security of a country and safeguarding the inviolability of the sea is an important manifestation of the sovereignty and dignity of a country. On the other hand, the ocean attracts mankind with its enormous resources such as marine living resources, marine energy, marine mineral resources, marine tourism resources, sea water resources, etc. The further development and utilization of marine resources have an increasingly important strategic significance for the long-term sustainable development of one country.

In order to develop marine high and new technology, develop marine resources, promote national economic construction and national defense construction, all kinds of marine vehicles must be vigorously developed. The ship is a very important kind of marine carrier, and the tracking of ship track is an important performance of the ship.

In the field of ship motion control, high precision of ship trajectory tracking control has been a key research. Heading control is the core of ship tracking. As a classical target tracking method, Line Of Sight (LOS) navigation algorithm uses the simple geometric relationship between point and point to judge visibility. It shows the advantages of simplicity and intuition, easy parameter setting and good convergence. The algorithm is independent of the dynamics controller and needs less design parameters. The expected heading's acquisition is only related to the position of ship and the given track, and the expected heading angle can be efficiently obtained.

Previous researchers use PID to control the heading[1-2]. PID control[3]can solve the trajectorytracking problem to some extent. But with actuator's amplitude limited, the integrator is prone to saturation and the heading appears oscillation and overshoot, which leads to low precision tracking and even instability of the system. Generalized Predictive Control (GPC) is a kind of predictive control method and developed with the study of adaptive control ${ }^{[4]}$. It is with three basic characteristics of model prediction, rolling optimization and feedback correction, also with an excellent control performance and robustness. Besides, on account ofthe use of the traditional parameter model (CARIMA model), the number of parameters is small and estimating parameters online becomes easy.

This paper proposes a method using GPC controller to control the expected heading angle obtained by LOS. The method is that the GPC controller is used to track the expected heading angle of LOS. By predicting the next output of few moments and optimizing the parameters of the controlled object in real time, the steering angle of the ship can be calculated. Finally, the ship can track with high precision.

\section{LOS navigation algorithm}

LOS navigation is reflected in the intuitive understanding of helmsman's steering and ship motion. The ship's heading command can be calculated by the target position. As long as following the heading command, the ship can keep tracking effectively[5]. InLOS navigation algorithm, ship navigates by calculating LOS angle[6-7].

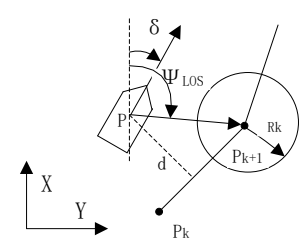

Figure 1. Schematic diagram of LOS navigation algorithm 
The angle is taken from positive direction of $x$ axis to the ray on the coordinate plane, which points from the origin of coordinates to the point $(x, y)$. The four quadrant tangent calculation is defined, so $\arctan (y, x) \in(-\pi, \pi)$ is the expected heading angle. As shown in Figure 1, the position of LOS is $P_{l o s}=\left[x_{l o s}, y_{l o s}\right]$ and the current position of the ship is $P=[x, y]$ in the earth coordinate system. The expected heading is obtained by using the ship's position and expected track, thus the ship's track is controlled indirectly. Then the LOS angle can be calculated by the following formula:

$$
\psi_{\text {los }}=\frac{\pi}{2}-\arctan \left(\frac{y_{l o s}-y}{x_{l o s}-x}\right)
$$

It is shown in Figure 2 that the position of the LOS is located between the preceding point $\left(x_{k}, y_{k}\right)$ and the last road point $\left(x_{k+1}, y_{k+1}\right)$, which represents two adjacent waypoint points. $(x, y)$ represents the ship's current position. Take $(x, y)$ as the center of the circle and $R$ as the radius ( $R=n L_{p p}$, the length of ship is $L_{p p}$, $n=2 \sim 5$ ) to draw an arc. When the ship is close to the track section, the arc will intersect the track at two points $A_{B}\left(t_{i}\right), A_{F}\left(t_{i}\right)$. The line of LOS is between the ship position $(x, y)$ and the point $A_{F}\left(t_{i}\right)$. And the expected heading is between the LOS and the horizontal coordinate $X$. According to the expected heading, tracking the virtual point can make the ship's track converge gradually to the given track. Under the action of the heading controller, the ship gradually converges to the expected track along the expected heading at a given speed.

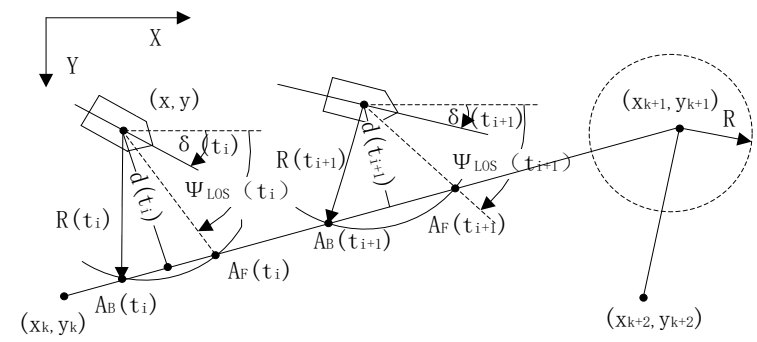

Figure 2.Schematic diagram of LOS navigation algorithm with fixed radius

When the ship has tracked along the current track, it needs a certain update principle to switch the expected track to the next track, so as to achieve the purpose of continuous tracking along the given track. When the controlled ship's position $(x, y)$ satisfies the following relations:

$$
\left(x_{k+1}-x\right)^{2}+\left(y_{k+1}-y\right)^{2} \leq R^{2}
$$

it is necessary to convert the current expected heading point into the next expected heading point. A new LOS angle is calculated by using the position of the ship and the data of the next expected heading point.

\section{High precision heading control}

\subsection{Establishment of ship heading model}

As formost of the ship motion and its control problems, the main variable is the movement speed $u$, traverse speed $^{v}$, yaw rate $r$. And the effects of heaving, pitching and rolling motion can be ignored. Therefore, it is considered that ship motion is in plane. It simplifies the problem of ship's motion to only three degrees of freedom[8]. Considering that the ship mass distribution is symmetric in $X Z$ plane, $I_{X Y}=I_{Y Z}=0$ and $y_{G}=0$, the ship motion equation is simplified as follows [9]:

$$
\left\{\begin{array}{c}
m\left(\dot{u}-r v-x_{G} r^{2}\right)=X \\
m\left(\dot{v}+r u-x_{G} \dot{r}^{2}\right)=Y \\
I_{z} \dot{r}+m x_{G}(\dot{v}+r u)=N
\end{array}\right.
$$

From the literature [10], it can be seen that the upper form can be expressed as the following simplified form:

$$
\left\{\begin{array}{c}
\dot{\eta}=J(\eta) v \\
M \dot{v}=-C(v) v-D(v) v+\tau+\tau_{E}
\end{array}\right.
$$

Where, $\eta=\left[\begin{array}{lll}x & y & \psi\end{array}\right]^{T}, v=\left[\begin{array}{lll}u & v & r\end{array}\right]^{T} . M$ is inertial parameter matrix,$C(v)$ is Coriolis centripetal force matrix, $D(v)$ Damping parameter matrix. Where $\tau=\left[\begin{array}{lll}\tau_{u} & 0 & \tau_{r}\end{array}\right]^{T}, \tau_{u}$ is propulsion, $\tau_{r}$ is yaw torque. Where $\tau_{E}=\left[\begin{array}{lll}\tau_{u E} & \tau_{v E} & \tau_{r E}\end{array}\right]^{T}, \tau_{u E} 、 \tau_{v E} 、 \tau_{r E}$ are the disturbances of sea acting on the advancing, transversal and yawing respectively.

Assuming that the initial state of a ship is uniform linear motion and the initial values of all variables is zero, the transfer function [10] of rudder angle to heading is as follows:

$$
G(s)=\frac{\psi(s)}{\delta(s)}=\frac{K\left(T_{3} s+1\right)}{s\left(T_{1} s+1\right)\left(T_{2} s+1\right)}
$$

As a ship is with large inertia, its dynamic characteristics are important in the low frequency range . Therefore, the Nomoto model[11]can be used to describe the upper form, which is to simplify three order model

$$
G(s)=\frac{\psi(s)}{\delta(s)}=\frac{K}{s(T s+1)}
$$

In this paper, the CARIMA model (also called ARIMAX model) is used to describe the Nomo to model:

$$
A\left(z^{-1}\right) \psi(k)=B\left(z^{-1}\right) \delta(k)
$$

where $z^{-1}$ is unit delay operator, $A\left(z^{-1}\right)$ and $B\left(z^{-1}\right)$ are polynomial coefficients where

$$
\begin{gathered}
A\left(z^{-1}\right)=1-\left(1+\frac{T}{T+T_{s}}\right) z^{-1}+\frac{T}{T+T_{s}} z^{-2}, \\
B\left(z^{-1}\right)=-\frac{K T_{s}^{2}}{T+T_{s}}
\end{gathered}
$$

and $T_{s}$ is the sampling period. 


\subsection{GPC control strategy}

As shown in Figure 3, the position and rudder angle of the ship are fed back to the LOS algorithm and the GPC controller respectively. The expected headingis calculated by the LOS, then the GPC controller tracks the heading angle to obtain a new command rudder angle. Ships can track continuously by repeating the above operation.

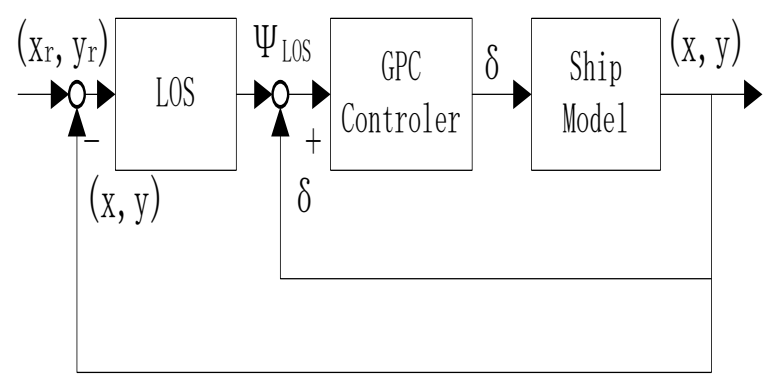

Figure 3. The schematic diagram of the control process

GPC is described by CARIMA model. The GPC algorithm proposed by [12] is used as the control step:

(1)According to the polynomial coefficients $A\left(z^{-1}\right)$, $B\left(z^{-1}\right)$ in the ship heading model, the predicted output is obtained as following:

$$
\begin{aligned}
\psi(t+1)= & \sum_{i=1}^{n} a_{1, i} \psi(t+1-i)+\sum_{i=0}^{m} b_{1, i} \Delta \delta(t-d-i) \\
& +\sum_{i=0}^{r} c_{1, i} e(t+1-i)
\end{aligned}
$$

here $\psi$ is the heading, $\delta$ is the rudder angle, $e$ is the external white noise, and $d+1$ is the pure delay of the system.

(2)The prediction output of $p$ step's heading is calculated by lower recursion. Where $\psi_{m}$ is form vector:

$$
\begin{aligned}
\psi_{m}(t+k) & =\sum_{i=1}^{n} a_{1, i} \psi_{m}(t+k-i) \\
& +\sum_{i=1}^{m} b_{1, i} \Delta \delta(t+k-d-1-i) \\
& +\sum_{i=1}^{r} c_{1, i} e(t+k-i)
\end{aligned}
$$

where $k=1, \cdots, p$, and $p$ is prediction step size

$$
\begin{aligned}
\Delta \delta(t+i) & =\left\{\begin{array}{ll}
0, & i \geq 0 \\
\Delta \delta(t+i), i<0
\end{array},\right. \\
e(t+i) & = \begin{cases}0, & i>0 \\
e(t+i), & i \leq 0\end{cases}
\end{aligned}
$$

(3)The actual operation requires that the heading approximates to the first-order inertial system and gradually approaches the set point $\psi_{r}$. Considering the delay of the system, the $p-d$ step reference trajectory is computed recursively by the lower form, $\psi_{r}$ is form vector, $\alpha$ is the diffusion coefficient,

$$
\left\{\begin{array}{l}
\psi_{r}(t+d)=\psi_{m}(t+d) \\
\psi_{r}(t+d+j)=\alpha \psi_{m}(t+d+j-1)+(1-\alpha) \psi_{s}
\end{array}\right.
$$

(4) The deviation of the rudder angle should be as small as possible in the heading keeping, and the rudder angle should not be changed too frequently. Therefore, objective function[13-14] is as following:

$$
J=\min \left\{\left(\psi_{r}-\psi\right)^{T}\left(\psi_{r}-\psi\right)+\lambda \Delta \delta^{T} \Delta \delta\right\}
$$

here $\lambda$ is weighted coefficient. The command angle of the $t$ moment is obtained by minimizing the objective function,

$$
\delta(k)=\delta(k-1)+\Delta \delta(k)
$$

where

$$
\Delta \delta(k)=(1,0, \cdots, 0)\left(G^{T} G+\lambda I\right)^{-1} G^{T}\left(\psi_{r}-\psi_{m}\right)
$$

Where

$$
G=\left[\begin{array}{cccc}
b_{1,0} & & & 0 \\
b_{2,0} & b_{1,0} & & \\
\cdots & \cdots & \ddots & \\
b_{p-d, 0} & b_{p-d-1,0} & \cdots & b_{1,0}
\end{array}\right]
$$

and

$$
b_{k, 0}=b_{1, k-1}+\sum_{j=1}^{k_{1}} a_{1, j} b_{k-j, 0}, \quad k=2,3, \cdots ; \quad k_{1}=\min \{k-1, n\}
$$

\section{Simulation and verification}

In simulation, the mariner model in GNC is used [15]. The length of ship is $\mathrm{L}=160$. The initial parameters are set. $\mathrm{n}=5$, the speed of ship is $20 \mathrm{kn}$, the speed of rudder angle is $5^{\circ} / \mathrm{s}$. The track is set from starting point $1(0,0)$, to the turning point $2(3000,3000)$, to the turning point 3 $(6000,2000)$, to the turning point $4(8000,8000)$, to the turning point $5(4000,6000)$, to the arrival point 6 $(2000,8000)$. Prediction step size is $p=10$, and the sampling period is $T_{s}=2$.

The simulation results are shown in Figure 4-6. Figure 4 is the expected track and the simulation track, in which the dashed line represents the expected track, and the solid part represents the simulation track. It can be seen from the image that the expected track is consistent with the simulated track. As is shown in Figure 5, there are four corners in the simulation process. The first, second, fourth turns is $\leq 90^{\circ}$ and the third turns is $>90^{\circ}$. According to Figure 4, it can be seen that the ship can track well at the first, second and fourth turn; the third expected heading angle changes greatly, which makes the heading's change of simulation delay greatly, but the change of expected track and simulation track is consistent. All angles on the trajectory can converge to the expected heading angle. 


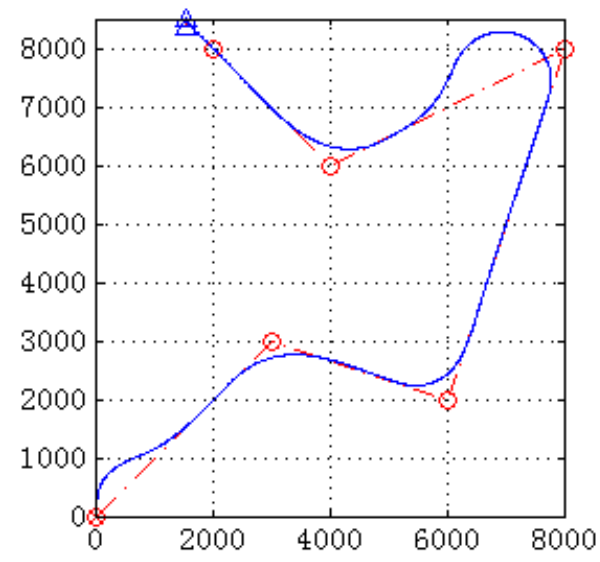

Figure 4. The expected track and the simulation track

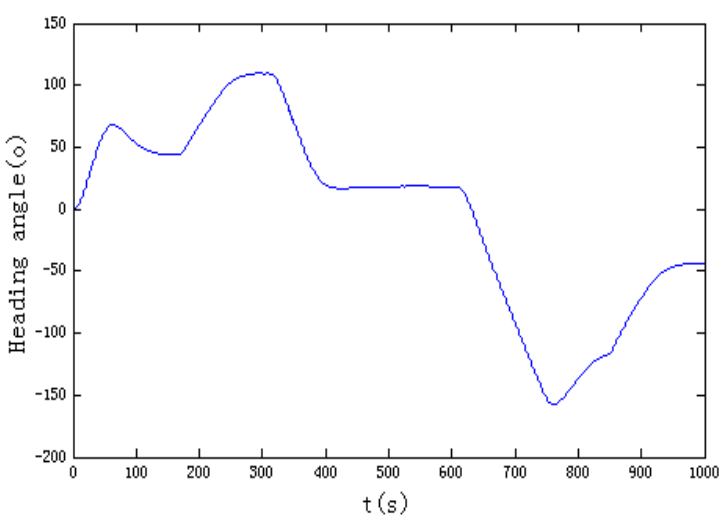

Figure 5. Heading angle curve

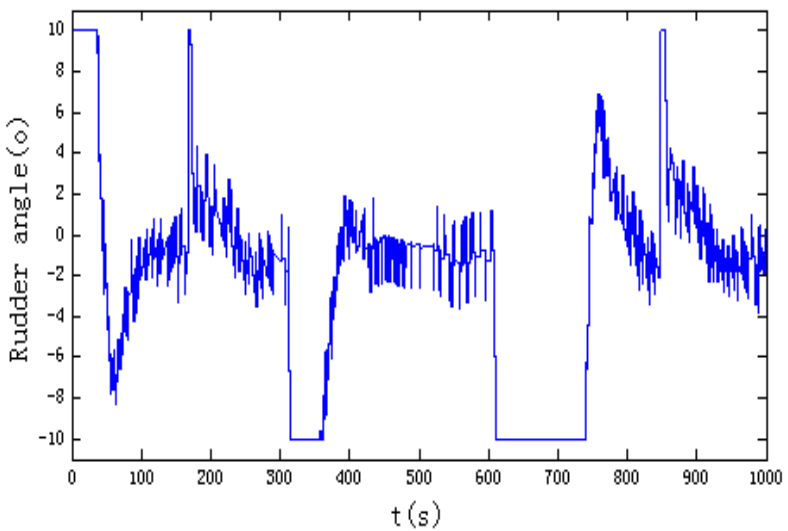

Figure 6. Rudder angle curve

In the straight path, two tracks almost coincide. In the case of small turning, the overshoot is very small, because the GPC has the characteristics of multi-step prediction, rolling optimization and feedback correction. In the case of large turning, the overshoot appears when switching is large. But it is in line with the actual situation when turning. The actual track is convergence to the expected track. Therefore, this method can make ship trajectory tracking highly precise.

\section{Conclusion}

This paper studies the ship's track tracking, according to this method, can achieve high-precision track tracking.

In this paper, LOS navigation algorithm and high precision heading tracking algorithm based on GPC are studied. LOS navigation algorithm can imitate the human visualization according to the setting. By means of judging the distance, the expected point is tracked, thus the expected heading angle can be obtained. The heading tracking algorithm designed in this paper can track well, and has good dynamic and static characteristics. Through theoretical deduction and simulation results, it is proved that the method can make ship track very effectively. Therefore, this research will make contributions to some insights and implication both theoretically and practically.Some physical experiments will be done in the future research to further verify the effectiveness of this method.

\section{References}

1. TIAN Yong,WANG Dan,PENG Zhou-hua,LIU Lu, Journal of Dalian Maritime University, 41,(4):1418,(2015)

2. HAN Peng,ZHOU Ze-cai,TANG Hao, Applied Science and Technology,44,(4):1-5, (2017)

3. ZENG Zhi-gang,YANG hai,HUANG Wang-jun, Research of Mobile Robot Navigation with SelfAdaptive Filtering and Fuzzy PID,22,(05):953957,(2015)

4. PENG Yan,WU Wei-qing,LIU Mei,USV Traching Control Based on Cascade GPC-PID,21,(5):245248,(2015)

5. Fossen T I, Breivik M, Skjetne R, University of Science and Technology(NTNU), NO.-7491,(2003)

6. Khaled N, Chalhoub N G, Nonlinear Dynamics, 73,(1-2):897-906, (2013)

7. Oh S R, Sun J, Ocean Engineering, 37,(2-3):289295(2010)

8. LIU Yang, LIU Mei-jie, Leader/Follower Formation Control of Underactuated Surface Ships, 20, (5):980-983,(2013)

9. Jia Xinle,Yang Yansheng, Chuan Bo Yun Dong Shu Xue Mo Xing-Ji LiJian Mo Yu Bian Shi Jian Mo(in chinese),(1999)

10. Zhang Y, Hearn G E, Sen P, Oceanic Engineering IEEE Journal of, 21,(4):513-527 (1996)

11. Nomoto $\mathrm{K}$, Taguchi K, Honda $\mathrm{K}$, et al.International Shipbuilding Progress, 4,(35):354-370 (2009)

12. Jin Yuanyu,Gu Xingyuan.Gai Jin De Guang Yi Yu Ce Kong Zhi Suan Fa(in chinese ),19, (3 ) :8-13 (1990)

13. HU Yao-hua, XU Su-wu, Control design for ship tracking based on prediction of desired headings, 27, (12):1641-1646,(2010)

14. HE Pei,QU Xiangju,Wu Zhe,Study of optimization method for flight path following,29,(7):598601,(2003)

15. Perez $\mathrm{T}$, Fossen $\mathrm{T}$ I,Modeling Identification \& Control, 28,(1):19-30(12) (2007) 